\title{
Experimental demonstration of all-optical PolMux RoF system with multiservice transmission
}

\begin{abstract}
In this paper, a Radio-over-Fiber (RoF) system is proposed by combining optical carrier suppression (OCS) and polarization multiplexing (PolMux) techniques in all-optical mode to enable multi-service transmission. Previous studies on RoF system only exploited multi-service transmission over low radio frequency (RF) oscillator-based PolMux, subcarrier multiplexing (SCM) and wavelength division multiplexing (WDM) techniques. Hence, herein, PolMux jointly with OCS techniques are exploited as a new medium of multiplexing in RoF system. Essentially, two contributions are unveiled, firstly the combination of all optical OCS and PolMux for RoF technology to enable multi-service transmission, and secondly an end-to-end all-optical transmission with the inclusion of wireless medium at millimeter wave (mmWave) frequency. The results indicate a highly reliable OCS-PolMux topology, where the EVM deterioration due to polarization leakage factor was only $1 \%$ to $2 \%$ for both services transmitted at the same frequency. Most importantly, the proposed system demonstrated an end-to-end system transmission with optical receiver power budget of up to $6 \mathrm{~dB}$.
\end{abstract}

Keyword: Optical carrier suppression; Polarization multiplexing; Radio-over-Fiber; Multiservice 